\title{
Analysis of microsatellite instability in CRISPR/Cas9 editing mice
}

\author{
Xueyun Huo ${ }^{a}$, Yating Du ${ }^{a}$, Jing Lu ${ }^{a}$, Meng Guo ${ }^{a}$ b, Zhenkun Li $^{a}$, Shuangyue Zhang ${ }^{a}$, \\ Xiaohong $\mathrm{Li}^{\mathrm{a}}$, Zhenwen Chen ${ }^{\mathrm{a}}$, Xiaoyan $\mathrm{Du}^{\mathrm{a}, \mathrm{b}, *}$ \\ a School of Basic Medical Science, Capital Medical University, Beijing 100069, China \\ b Department of Laboratory Animal, Capital Medical University, Beijing 100069, China
}

\section{A R T I C L E I N F O}

\section{Article history:}

Received 11 May 2016

Received in revised form 26 February 2017

Accepted 26 February 2017

Available online 28 February 2017

\section{Keywords:}

CRISPR/Cas9

Microsatellite instability (MSI)

C57BL/6J mice

\begin{abstract}
A B S T R A C T
Clustered regularly interspaced short palindromic repeats (CRISPR)/CRISPR- associated (Cas) protein 9 system is a novel and powerful tool which is widely used for genome editing. CRISPR/Cas9 is RNA-guided and can lead to desired genomic modifications. However, whether the CRISPR/Cas9-mediated genome editing causes genomic alterations and genomic instability, such as microsatellite instability (MSI), is still unknown. Here we detected MSI in $21 \mathrm{CRISPR/Cas9} \mathrm{mouse} \mathrm{strains} \mathrm{using} \mathrm{a} \mathrm{panel} \mathrm{of} 42$ microsatellite loci which were selected from our previous studies. Surprisingly, MSI occurrence was common in CRISPR/Cas9 modified genome, and most of the strains (19/21, 90.5\%) examined showed MSI. Of 42 loci examined, 8 loci $(8 / 42,19.05 \%)$ exhibited MSI in the Cas9 editing mice. The Ttll9 $(4 / 42,9.5 \%)$ were the most unstable strains, and D10Mit3 and D10Mit198 (9/21, 42.9\%) were considered to be the most "hot" loci in the Cas9 strains we tested. Through analyzing the mutation of microsatellite loci, we provide new insights into the genomic alterations of CRISPR/Cas9 models and it will help us for a better understanding of this powerful technology.
\end{abstract}

(c) 2017 Published by Elsevier B.V.

\section{Introduction}

Microsatellites are short tandem repeats (STRs) of DNA sequences that consist of 1-6 base pairs (bp) units. STRs are highly polymorphic, and abundantly and randomly distributed throughout eukaryotic genomes [1]. Because of the special structure of microsatellite sequence, the DNA polymerase is vulnerable to slippage and lead to indels in repetitive sequence tracts during DNA replication, which is named as microsatellite instability (MSI). Therefore, microsatellite loci are recognized as "sentinel" DNA or as hotspots that indicate genomic changes [2].

The clustered regularly interspaced short palindromic repeat (CRISPR)-associated Cas9 system has recently emerged as a novel and powerful genome editing tool [3-5]. This system is a RNAguided genetic silencing system in bacteria and archaea, and functions as a prokaryotic immune system fighting against foreign nucleic acids carried by invading viruses or other pathogens [3,6-8]. This genomic editing system has been successfully adopted into mammalian cells with codon optimization of the Cas9 coding sequence [5,9-11]. Once inside the cells, the Cas9 nuclease is directed to a targeted genomic locus by synthetic guide RNA

\footnotetext{
* Corresponding author.

E-mail address: duduyan@ccmu.edu.cn (X. Du)
}

(sgRNA), creating a DNA double strand breaks (DSBs) which are subsequently repaired by either non-homologous end joining (NHEJ) or homology-directed repair (HDR). The NHEJ-mediated repair often causes small insertions or deletions (indels), leading to frameshift mutations; whereas the HDR usually enables the replacement of the endogenous target DNA sequence with any desired homologous DNA template sequence [12,13]. Cas9-based gene editing technology is broadly applicable than other genome engineering systems, such as zinc finger nucleases (ZFNs) and transcription activator-like effector nucleases (TALENs), with simple and speedy design and construction of the CRISPR/Cas9 systems [3,7]. It has dramatically improved the generation efficiency for many genetically modified models, with many lines of single and/or multiple gene knockout, or transgenic models have been successfully generated in mouse $[4,14,15]$, rat [16], and human cell lines [3,5].

The NHEJ DNA repair pathway is error prone, and may introduces insertions and deletions (INDELs); thus, Cas9 editing would induce genomic instability. We have found that genome instability can be induced by different types of genetic modification of mouse genome, which include conventional gene knockout by homologous recombination (referred to KO mice), transgenic random insertion of external DNA fragments into the host genome (referred to $\mathrm{Tg}$ mice), and spontaneous induction of mutagenesis by in vivo administration of mutagenic chemicals. Microsatellite instability (MSI) is a highly mutable phenotype caused by DNA mis- 
Typy I (D14Nds1)

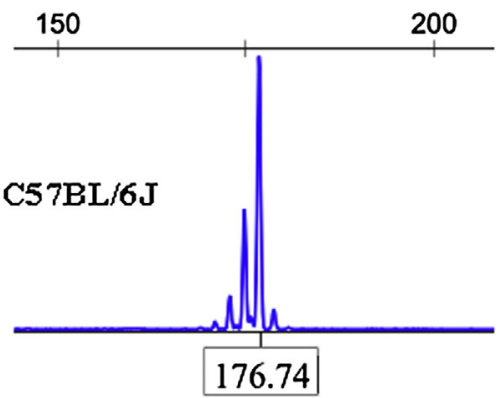

150

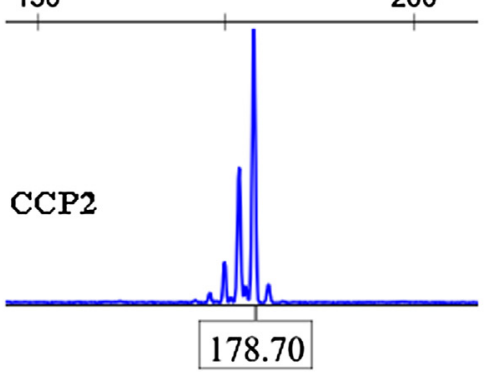

Type II (D3Mit22)
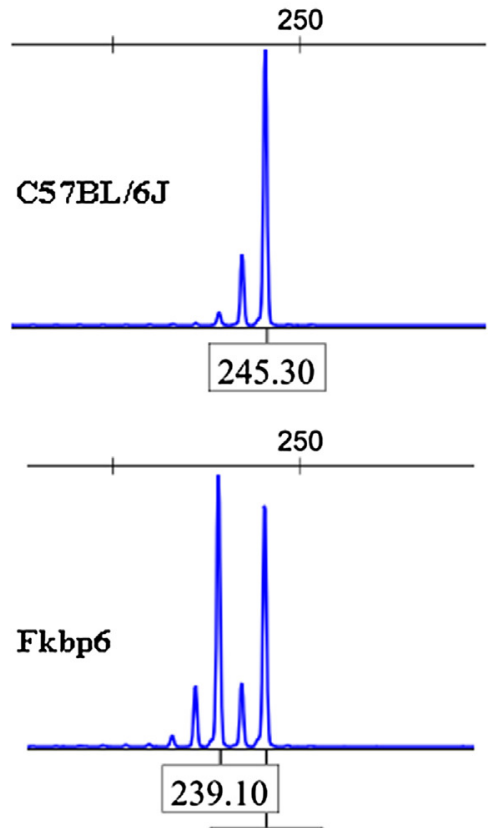

245.21

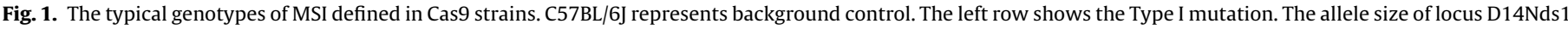

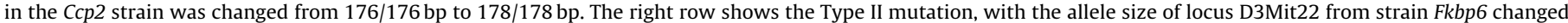
from $245 / 245$ bp to $239 / 245$ bp.

Table 1

The MSIs at 8 loci detected in 21 Cas9 strains.

\begin{tabular}{|c|c|c|c|c|c|c|c|c|c|c|}
\hline Strains & D10Mit3 & D10Mit198 & D3Mit17 & D3Mit22 & D11Mit2 & D18Mit19 & D14Nds1 & D13Mit3 & Total & Frequency \\
\hline Ttll9 & $-8^{*}$ & $-2 *$ & $-4^{*}$ & & $-12 ;+8 *$ & & & & 4 & $9.5 \%$ \\
\hline Fkbp6 & $-4 *$ & $-2 *$ & & $-6 *$ & & & & & 3 & $7.1 \%$ \\
\hline$B b c 3$ & $-4 \#$ & & & $-6^{*}$ & $-12 *$ & & & & 3 & $7.1 \%$ \\
\hline Ttll4 & $-6 *$ & & & $-6 *$ & & & & $-4^{*}$ & 3 & $7.1 \%$ \\
\hline $\mathrm{Nmi}$ & $-2 \#$ & & & & $-12 *$ & & & & 2 & $4.8 \%$ \\
\hline$K d m 2 b$ & $-6 *$ & $-2 *$ & & & & & & & 2 & $4.8 \%$ \\
\hline Ttll6 & $-6 *$ & & & & $-12 *$ & & & & 2 & $4.8 \%$ \\
\hline Zfp64 & $-4^{*}$ & & & $-6^{*}$ & & & & & 2 & $4.8 \%$ \\
\hline Ccp2 & & $-2 *$ & & & & & $+2 \#$ & & 2 & $4.8 \%$ \\
\hline Ccp4 & & $-2 *$ & $-4^{*}$ & & & & & & 2 & $4.8 \%$ \\
\hline Gipc2 & & $-2^{*}$ & $-4^{*}$ & & & & & & 2 & $4.8 \%$ \\
\hline Gm2694 & $-4^{*}$ & & $-4^{*}$ & & & & & & 2 & $4.8 \%$ \\
\hline Ссp3 & & $-2 *$ & & & & & & & 1 & $2.4 \%$ \\
\hline wipi3 & & $-2^{*}$ & & & & & & & 1 & $2.4 \%$ \\
\hline Slc38a4 & & $-2 *$ & & & & & & & 1 & $2.4 \%$ \\
\hline Them2 & & & & & & $-2 *$ & & & 1 & $2.4 \%$ \\
\hline Scarb2 & & & $-4^{*}$ & & & & & & 1 & $2.4 \%$ \\
\hline Fh15 & & & $-4 \#$ & & & & & & 1 & $2.4 \%$ \\
\hline Nlp14 & & & $-4^{*}$ & & & & & & 1 & $2.4 \%$ \\
\hline Ccp5 & & & & & & & & & 0 & $0.0 \%$ \\
\hline wipi1 & & & & & & & & & 0 & $0.0 \%$ \\
\hline Sum & 9 & 9 & 7 & 4 & 4 & 1 & 1 & 1 & 36 & \\
\hline Frequency & $42.9 \%$ & $42.9 \%$ & $33.3 \%$ & $19.0 \%$ & $19.0 \%$ & $4.8 \%$ & $4.8 \%$ & $4.8 \%$ & & \\
\hline
\end{tabular}

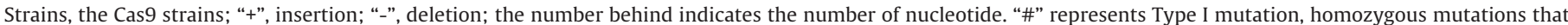
involved either a deletion or an insertion; “*” represents Type II mutation, heterozygous mutations in which the mutation only presented at a given allele of a locus.

match repair impairment. We have founded enhanced MSI in KO, $\mathrm{Tg}$, spontaneously mutated, and the ENU-treated and MNU-treated mice [17-19]. However, whether CRISPR/Cas9 genomic editing can also induce unpredictable genomic polymorphisms such as MSI is currently unknown. It is well known that MSI is highly linked to tumorigenesis $[20,21]$. To evaluate the potential risk of genomic instability by CRISPR/Cas9 genome engineering, we have examined the MSI occurrence in 21 CRISPR/Cas9 mouse strains (referred to Cas9 strains) at 42 "hotspot" microsatellite loci that have been repeatedly selected in our previous studies [19]. We fique is used to modify the target gene activity. 


\section{Materials and methods}

\subsection{Sample collection}

The genomic DNAs from C57BL/6J inbred wild-type controls and 21 genetically modified C57BL/6J mouse strains created by CRISPR/Cas9 technology were obtained from the laboratory of Dr. Yong Tian at the Institute of Biophysics, Chinese Academy of Sciences.

The targeted gene or edited locus for each KO mouse strain are shown in Table 1. Some of the deficient mouse strains has been published and the CRISPR-Cas9 editing approaches as described [22]. The parental mice for all of the KO mice used in the present study were the inbred C57BL/6J strains in which the individual's genome was highly homozygous and could be regarded as genetically identical.

\subsection{Microsatellite analysis}

Forty-two microsatellite loci that had been previously identified in our studies of the genetically modified and chemical treated mice were used in this study. The forward primer for each locus was tagged at the $5^{\prime}$ end with one of FAM, HEX or TAMRA fluorescent markers separately. The PCR amplification was carried out in a total volume of $20 \mu \mathrm{L}$, consisted of $2 \mu \mathrm{L}$ of $10 \times$ buffer, $0.5 \mu \mathrm{mol} / \mathrm{L}$ of each primer, $125 \mu \mathrm{mol} / \mathrm{L}$ dNTP $(4 \times), 1.0 \mathrm{U}$ of Taq DNA polymerase, $1.5-2.5 \mathrm{mmol} / \mathrm{L} \mathrm{MgCl}_{2}$, and $100 \mathrm{ng}$ of template DNA. The PCR reaction was performed in a gradient thermal cycler (BIO-RAD Inc. ALS1296) in accordance with the protocol of pre-denaturation at $94^{\circ} \mathrm{C}$ for $5 \mathrm{~min}$; 35 cycles of denaturation at $94^{\circ} \mathrm{C}$ for $30 \mathrm{~s}$, annealing at the optimized temperature for each microsatellite for $30 \mathrm{~s}$, and extension at $72{ }^{\circ} \mathrm{C}$ for $30 \mathrm{~s}$, followed by a final extension at $72{ }^{\circ} \mathrm{C}$ for $5 \mathrm{~min}$. The amplification products were evaluated on $2 \%$ agarose gels stained with ethidium bromide and visualized using a UV transilluminator (BIO-RAD Inc. Gel Doc ${ }^{\mathrm{TM}} \mathrm{XR}+$ ). When the PCR products were amplified successfully and correctly, the corresponding samples were carefully stored at $4{ }^{\circ} \mathrm{C}$ for STR scanning and DNA sequencing.

For STR analysis, $1 \mu \mathrm{L}$ of the fluorescent labeled PCR products from samples, containing a mixture of three microsatellite PCR products labeled individually with FAM, HEX, and TAMRA at a ratio of $1: 2: 3$, respectively, was combined with $25 \mu \mathrm{L}$ of formamide; and the combined solution was visualized using capillary electrophoresis on an ABI-3730XL DNA Analyzer system (PE Biosystems, USA). The peak height of the wave was determined using GeneMarker version 1.75. For sequence analysis of the MSI locus after STR scanning, PCR amplification was performed again in a total volume of $50 \mu \mathrm{L}$, and the PCR products were purified using an ABI BigDye Terminator v3.1 Cycle Sequencing Kit, cloned into the PMD18-T Vector (TAKARA), and then sequenced using an ABI 3730XL DNA Sequencer.

\subsection{Statistical analysis}

Statistical analysis was performed using SPSS 16.0 (SPSS Inc., USA). The incidence comparison of different groups was conducted using a chi-square test. A $p$ value $<0.05$ was considered statistically significant.

\section{Results}

\subsection{MSI analysis of the CRISPR/Cas9 edited mice by STR scanning}

MSI analysis of 42 microsatellite loci was performed on 21 Cas9 strains using STR scanning, and 2 wild-type C57BL/6J mice were used as blank control. After detailed analysis of the STR data, we
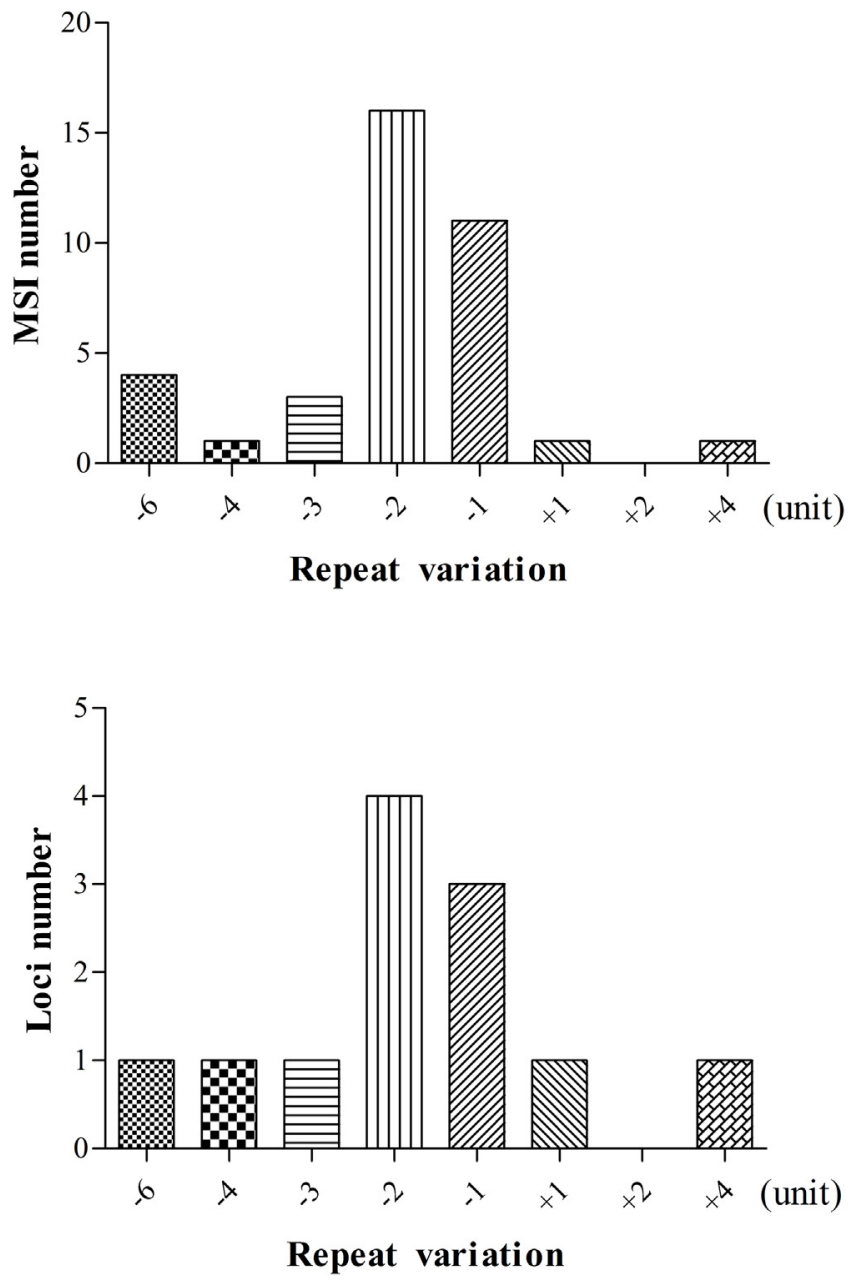

Fig. 2. The mutation patterns of MSI detected in Cas9 strains. "+”, insertion; “-”, deletion; the number behind indicates the number of repeat unit. The MSI number of each mutation pattern showed that the most frequency pattern are 2 and 1 unit deletion. The loci number of each mutation pattern demonstrated that 2 and 1 unit deletion are more common in different loci.

identified 36 MSI events that were distributed in 8 loci, representing an approximately $19.0 \%$ of loci (8/42) showing MSI. Strikingly, there were 19 out of the 21 Cas9 strains (19/21, 90.5\%), with exception of Wipi1 and Ccp5 strains, exhibiting MSI when compared to the wild-type controls. The names of the MSI positive loci and the Cas9 strains that carry the MSI are shown in Table 1.

From this analysis, we found that the most unstable strains were Ttll9, which exhibited four microsatellite loci (4/42, 9.5\%) carrying MSI. The next unstable strains were Fkbp6, Bbc3 and Ttll4, with each strain generating MSI at three loci $(3 / 42,7.1 \%)$. There were eight strains, Nmi, Kdm2b, Ttll6, Zfp64, Ccp2, Ccp4, Gipc2 and Gm2694, showing MSI at two loci (2/42, 4.8\%). The least unstable strains were Ccp3, Wipi3, Slc38a4, Them2, Scarb2, Fh15 and Nlp14, showing MSI at only one loci $(1 / 42,2.4 \%)$.

Among the affected loci, D10Mit3 and D10Mit198 were the most vulnerable loci with MSI found in 9 out of 21 Cas9 strains, representing roughly $(9 / 21,42.9 \%)$ of CRISPR/Cas9 edited strains generating MSI at these loci; and the next most affected loci were the D3Mit17, appearing in 7 strains $(7 / 21,33.3 \%)$ carrying MSI. The mild-affected loci, were D3Mit22 and D11Mit2, showing MSI in 4 strains $(4 / 21$, 19.0\%); and followed by the least affected loci, D18Mit19, D14Nds1, and D13Mit3, which exhibited MSI only in one strain (1/21, 4.8\%).

The MSIs detected in the present study can be classified into two typical patterns (Fig. 1). The Type I MSI was a homozygous muta- 


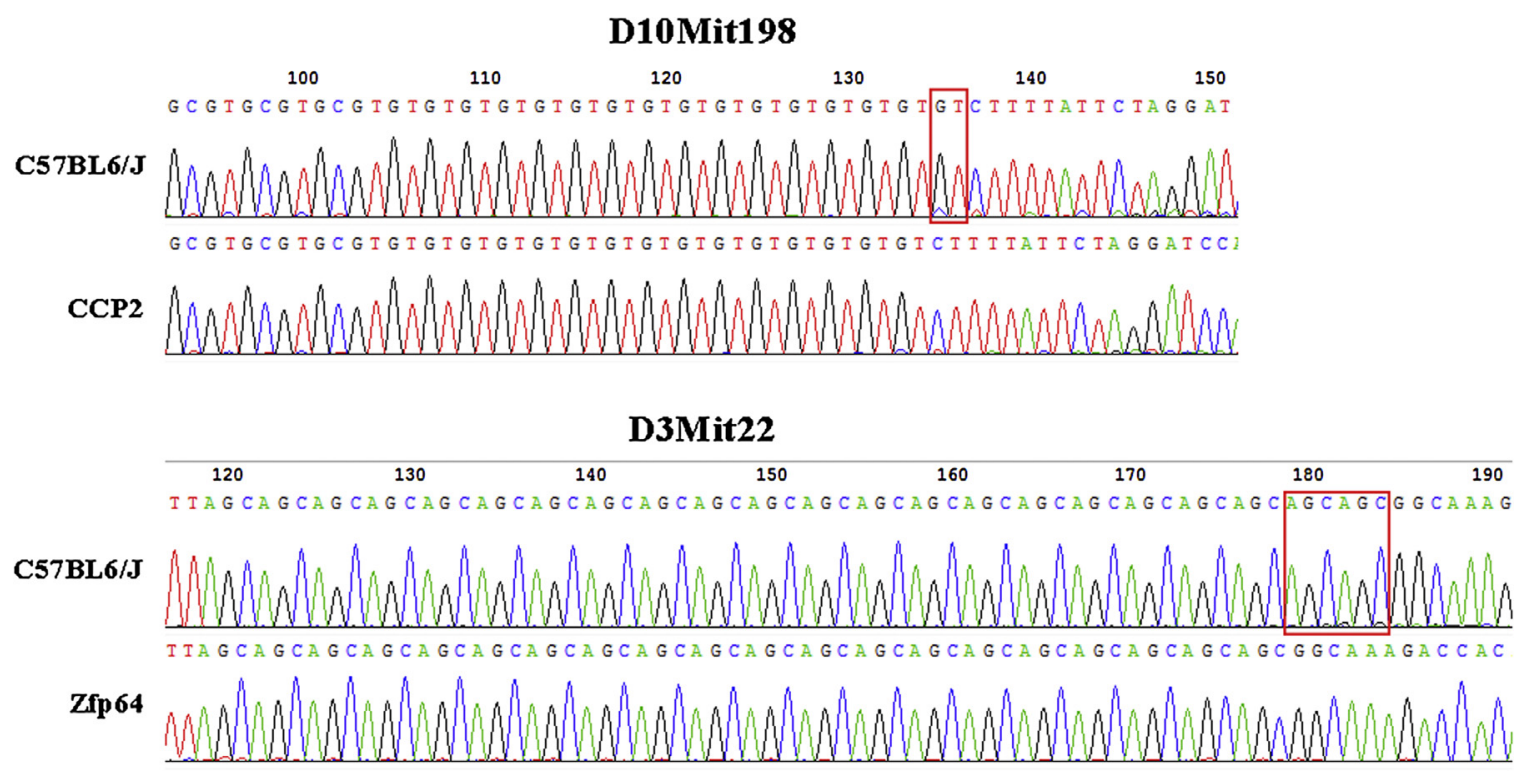

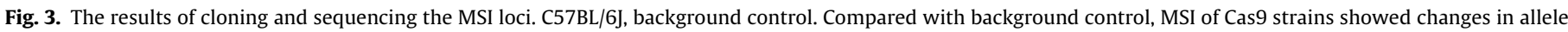
size. D10Mit198 showed the loss of an (GT) repeat unit; D3Mit22 showed the deletion of two (AGC) repeat unit.

tion in which the two alleles of the homozygous locus showed an identical variation involving either a deletion or an insertion; and the Type II was a heterozygous mutation in which the mutation occurred at one specific allele within the locus. Among $8 \mathrm{MSI}$ loci, D14Nds1 $(12.5 \%, 1 / 8)$ was the Type I mutation with a 2-bp addition, and D3Mit22, D11Mit2, D10Mit198, D18Mit19 and D13Mit3 $(62.5 \%, 5 / 8)$ were the Type II MSIs, with mutations of either $2-12 \mathrm{bp}$ deletion or $4-8$ bp insertion. Interestingly, the loci D10Mit3 and D3Mit17 could be identified by either type of mutations $(25.0 \%$, $2 / 8$ ) (Table 1 ). Our data indicated that majority of MSIs was characterized as the Type II mutation (32/36, 88.9\%), and only 4 of 36 MSIs (11.1\%) were identified as Type I mutation $(p<0.0001)$ (Table 1$)$.

Analysis of the variation length among 36 MSI mutations indicated that majority (35/36) of these MSIs carried one single defined length of variation, with one exception that D11Mit2 in Slc38a4 mice contained two length variation $(-12 \mathrm{bp}$ and $+8 \mathrm{bp}$ ) (Table 1$)$. Among the 37 indels, the number of deletions $(35 / 37,94.6 \%)$ was remarkably higher than insertions $(2 / 37,5.4 \%)(p<0.0001)$. The core sequence of most of the loci were characterized by dinucleotide repeats, except that D3Mit22 is the locus with a trinucleotide repeat. Two most frequent mutation patterns were characterized as 2 unit deletions insertions $(16 / 37,43.2 \%), 1$ unit deletions $(11 / 37$, 29.7\%). The most popular mutation patterns in different loci were also 2 and 1 unit deletions which detected in 4 and 3 loci respectively (Fig. 2).

\subsection{MSI analysis by clone sequencing in Cas9 strains}

To obtain the detailed sequence information from all the MSIs identified by STR scanning, we cloned the PCR products amplified from all of the 36 MSI loci into the pMD18-T Vector (TAKARA). The PCR products in the plasmid were subjected to sequencing analysis. The PCR products from these loci in the wild-type C57BL/6J mice were used as control for effectively and accurately analysis of the MSI sequences in the Cas9 strains. The detailed sequence variation information is exampled in Fig. 3. These DNA sequencing results further verified the MSI identity detected by STR scanning in the Cas9 strains. This result suggests that MSI identification by DNA cloning and sequencing is consistent with analysis by STR scanning method.

\section{Discussion}

We have analyzed, for the first time, the MSI occurrence in CRISPR/Cas9 generated mouse strains. The 42 "hotspot" microsatel-

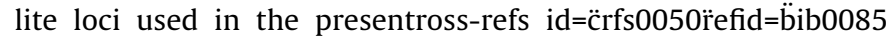

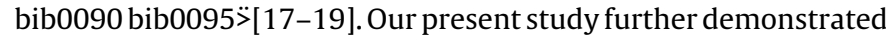
that CRISPR/Cas9-mediated genome editing can also trigger MSI, suggesting the potential risks existed when the CRIPSR/Cas9 technique is used to modify the target gene activity, especially in the human cell lines or embryonic cells, or used for therapeutic applications.

Off-target effects are the unwanted mutations caused by CRISPR/Cas9 nucleases on DNA sequences that are identical or highly homologous to the target DNA sequences. These off-target mutations can result in cell death or transformation, and confound research experiments; and therefore, more and more efforts have been paid to reduce genome-wide off-target effects $[5,23,24]$. In the present study, we used STR scanning and direct DNA sequencing to screen MSI on 42 precisely selected microsatellite loci, and found that the CRISPR/Cas9 mediated genome editing was not exclusively restricted to the targeted gene loci and off-target sites, but also occurred in other sites of the genome.

By using the same panel of microsatellite loci, we have previously identified spontaneously occurred MSI in 11 out of $29(37.9 \%)$ KO mouse strains [17]. Strikingly, the frequency of MSI-positive Cas9 strains $(19 / 21,90.5 \%)$ was remarkably higher than that of the KO mouse strains $(p=0.0003)$. The MSI frequency in the Cas9 strains was $4.1 \%$ [36 MSI/(42 loci $\times 21$ strains $)$, which was also significantly higher than that in the KO mouse strains [0.9\%, 12 $\mathrm{MSI} /(42$ loci $\times 29$ strains $)](p<0.0001)$. Among the MSI-positive KO mouse strains, two $(2 / 11,18.1 \%)$ harbored more than one MSI event and the rest strains had only one MSI event. Whereas, in the 19 MSI positive Cas9 strains, twelve $(12 / 19,63.2 \%)$ harbored more than one MSI event $(p=0.0259)$ (Table 2$)$. Besides, we found that the MSI frequency in the MNU and ENU treated strains was $0.9 \%$ and $0.1 \%$, which was also significantly lower than that in the Cas9 strains $(p<0.0001)$. Our results suggest that MSI occurrence is more common in the CRISPR/Cas9 modified mouse strains than in the conventional $\mathrm{KO}$ and chemical mouse strains. This difference in MSI frequency may be caused by the different experimental strategies that these approaches adopted. It is well known that the traditional 
Table 2

The MSI-positive status in KO and Cas9 mouse models.

\begin{tabular}{llll}
\hline & KO & Cas9 & $p$-value \\
\hline MSI+ strains & $11 / 29(37.9 \%)$ & $19 / 21(90.5 \%)$ & 0.0003 \\
MSI+ strains $(\geq 2)$ & $2 / 11(18.1 \%)$ & $12 / 19(63.2 \%)$ & 0.0259 \\
MSI frequency & $0.9 \%$ & $4.1 \%$ & $<0.0001$ \\
\hline
\end{tabular}

MSI+ strains, strains found with MSI event; MSI+ strains $(\geq 2)$, strains found with more than one MSI events.

KO is carried out by replacement of a targeted gene with a large external DNA fragment to ensure the homologous recombination; whereas, the Cas9 nuclease-mediated genome editing depends on the guidance by a 20-nt short homologous gRNA sequence. Obviously, the recognition accuracy of CRISPR/Cas9 is far less than the conventional $\mathrm{KO}$, and may lead to relative high risk of off-target mutations. Consequently, these unwanted genomic variations in CRISPR/Cas9 may further trigger genome instability causing MSI. Compared with Cas9 modified mouse strains, the genomic modification of chemical mouse strains are induced by mutagenic agent in a random process and the mutations need time to accumulate, that maybe a reason for the lower MSI frequency in MNU and ENU treated strains.

Of the unstable loci we identified in the Cas9 strains, D10Mit3 and D10Mit198 were the most vulnerable locations for MSI (9/21, 42.9\%) compared to other loci. Interestingly, we previously reported that this locus also generated MSI with high frequency in the Tg and MNU treated mouse strains. Among these hotspots, D10Mit198, D11Mit2 and D14Nds1 were found with MSI in the Cas9, Tg, and MNU treated mouse strains; D3Mit22 and D13Mit3 were shared among Cas9, Tg, and KO strains; D14Mit102 carried MSI simultaneously in the Tg, KO, MNU and ENU strains; D3Mit278 were shared among Tg, KO and MNU strains; D15Mit5 were found with MSI in the Tg, MNU and ENU strains. These shared loci might be MSI "hotspots" in genetic modified mouse strains although they were incomparable to those in other species or human beings [17-19] (Table S1).

In this study, the most frequently unstable Cas9 strains were Ttll9 (4/42, 9.5\%), suggesting that the Cas9-based editing of some target loci intends more likely to induce MSI, which, of course, does not exclude a coincidence due to the limitation of the loci we selected. Different Cas9 targets induce different MSI rate maybe due to some of the sgRNAs have more off-target effects, because the sequence of each sgRNA is quite different. Besides, the differences of spatial position and functions of different Cas9 target genes maybe another reason that induce different MSI rate.

Through analyzing the features of MSI occurrence, we found that the number of type II mutation (32/36, 88.9\%) was markedly greater than type I mutation (11.1\%). MSI is a stochastic process, it is theoretically reasonable that homozygous slippage of a locus is much less frequent than a heterozygous mutation at single allele. The instability was preferentially driven by deletion $(94.6 \%)$ than by insertion (5.4\%), suggesting that these microsatellite loci are more susceptible to deletion mutations in the Cas9 strains. Besides, mutation pattern also showed a length preference, the 2 and 1 unit deletions seemed to be more popular at different loci for they presented in 4 and 3 loci respectively.

Consistent with our previous report $[17,19]$, the present data imply that the locations of MSI have no obvious correlation with the edited genes, and they are often located in different chromosomes. MSI is considered to be one of major forms of genomic instability [25], and the high level of MSI has been considered to contribute to tumorigenesis in several cancers [26-28]. Many studies have demonstrated that MSI results from the deficiency in DNA mismatch repair (MMR) systems [29]. But, the mechanism underlying how the CRISPR/Cas9 system triggers MSI remains to be investigated in the future.

Our study provides new evidence for safety uses of the CRISPR/Cas9 system, and will be beneficial for designing therapeutic strategies for clinical applications. The high frequency of the off-target mutations in the CRISPR/Cas9 engineered cells has recently drawn risk concerns $[24,30]$. High frequency, and unpredictable occurrence of MSIs in the CRISPR/Cas9 modified mice set alerts for safety uses of the conventional CRISPR/Cas9 system. Development of high-fidelity CRISPR/Cas9 to overcome the genome-wide instability is therefore encouraged.

\section{Conflict of interest statement}

None declared.

\section{Acknowledgements}

We thank Dr. Yong Tian from the Institute of Biophysics, Chinese Academy of Sciences for kindly providing the CRISPR/Cas9 editing mouse strains.

This study was funded by Key Projects in the National Science \& Technology Pillar Program (Nos. 2015BAI09B01; 2015BAI07B01), the National Science Foundation of China (No. 31372272) and the Beijing Natural Science Foundation (No. 7141002).

\section{Appendix A. Supplementary data}

Supplementary data associated with this article can be found, in the online version, at http://dx.doi.org/10.1016/j.mrfmmm.2017. 02.003 .

\section{References}

[1] D. Tautz, M. Renz, Simple sequences are ubiquitous repetitive components of eukaryotic genomes, Nucleic Acids Res. 12 (1984) 4127-4138.

[2] J. Klapacz, G.M. Lingaraju, H.H. Guo, D. Shah, A. Moar-Shoshani, L.A. Loeb, L.D. Samson, Frameshift mutagenesis and microsatellite instability induced by human alkyladenine DNA glycosylase, Mol. Cell 37 (2010) 843-853.

[3] P. Mali, L. Yang, K.M. Esvelt, J. Aach, M. Guell, J.E. DiCarlo, J.E. Norville, G.M Church, RNA-guided human genome engineering via Cas9, Science 339 (2013) 823-826.

[4] T. Horii, I. Hatada, Production of genome-edited pluripotent stem cells and mice by CRISPR/Cas [Review], Endocr. J. 63 (2016) 213-219.

[5] L. Cong, F.A. Ran, D. Cox, S. Lin, R. Barretto, N. Habib, P.D. Hsu, X. Wu, W. Jiang, L.A. Marraffini, F. Zhang, Multiplex genome engineering using CRISPR/Cas systems, Science 339 (2013) 819-823.

[6] D. Bhaya, M. Davison, R. Barrangou, CRISPR-Cas systems in bacteria and archaea: versatile small RNAs for adaptive defense and regulation, Annu. Rev. Genet. 45 (2011) 273-297.

[7] M. Jinek, K. Chylinski, I. Fonfara, M. Hauer, J.A. Doudna, E. Charpentier, A programmable dual-RNA-guided DNA endonuclease in adaptive bacterial immunity, Science 337 (2012) 816-821.

[8] J.E. Garneau, M.E. Dupuis, M. Villion, D.A. Romero, R. Barrangou, P. Boyaval, C. Fremaux, P. Horvath, A.H. Magadan, S. Moineau, The CRISPR/Cas bacterial immune system cleaves bacteriophage and plasmid DNA, Nature 468 (2010) $67-71$.

[9] S.W. Cho, S. Kim, J.M. Kim, J.S. Kim, Targeted genome engineering in human cells with the Cas9 RNA-guided endonuclease, Nat. Biotechnol. 31 (2013) 230-232.

[10] P. Essletzbichler, T. Konopka, F. Santoro, D. Chen, B.V. Gapp, R. Kralovics, T.R. Brummelkamp, S.M. Nijman, T. Burckstummer, Megabase-scale deletion using CRISPR/Cas9 to generate a fully haploid human cell line, Genome Res. 24 (2014) 2059-2065.

[11] X. Chen, M. Li, X. Feng, S. Guang, Targeted chromosomal translocations and essential gene knockout using CRISPR/Cas9 technology in caenorhabditis elegans, Genetics 201 (2015) 1295-1306.

[12] N. Savić, G. Schwank, Advances in therapeutic CRISPR/Cas9 genome editing, Transl. Res. 168 (2016) 15-21.

[13] D.E. Barnes, Non-homologous end joining as a mechanism of DNA repair, Curr. Biol. 11 (2001) R455-R457.

[14] U. Elling, J. Taubenschmid, G. Wirnsberger, R. O’Malley, S.P. Demers, Q. Vanhaelen, A.I. Shukalyuk, G. Schmauss, D. Schramek, F. Schnuetgen, H. von Melchner, J.R. Ecker, W.L. Stanford, J. Zuber, A. Stark, J.M. Penninger, Forward 
and reverse genetics through derivation of haploid mouse embryonic stem cells, Cell Stem Cell 9 (2011) 563-574.

[15] H. Wang, H. Yang, C.S. Shivalila, M.M. Dawlaty, A.W. Cheng, F. Zhang, R. Jaenisch, One-step generation of mice carrying mutations in multiple genes by CRISPR/Cas-mediated genome engineering, Cell 153 (2013) 910-918.

[16] Y. Shao, Y. Guan, L. Wang, Z. Qiu, M. Liu, Y. Chen, L. Wu, Y. Li, X. Ma, M. Liu, D Li, CRISPR/Cas-mediated genome editing in the rat via direct injection of one-cell embryos, Nat. Protoc. 9 (2014) 2493-2512.

[17] B. Zuo, X. Du, J. Zhao, H. Yang, C. Wang, Y. Wu, J. Lu, Y. Wang, Z. Chen, Analysis of microsatellite polymorphism in inbred knockout mice, PLoS One 7 (2012) e34555.

[18] X. Huo, S. Zhang, Z. Li, J. Gao, C. Wang, C. Li, M. Guo, X. Du, Z. Chen, Analysis of the relationship between microsatellite instability and thymic lymphoma induced by N-methyl-N-nitrosourea in C57BL/6J mice, Mutat. Res. 771 (2015) $21-28$.

[19] X. Du, J. Cui, C. Wang, X. Huo, J. Lu, Y. Li, Z. Chen, Detected microsatellite polymorphisms in genetically altered inbred mouse strains, Mol. Genet. Genomics 288 (2013) 309-316.

[20] K. Imai, H. Yamamoto, Carcinogenesis and microsatellite instability: the interrelationship between genetics and epigenetics, Carcinogenesis 29 (2008) 673-680.

[21] S.N. Thibodeau, G. Bren, D. Schaid, Microsatellite instability in cancer of the proximal colon, Science 260 (1993) 816-819.

[22] P. Xia, B. Ye, S. Wang, X. Zhu, Y. Du, Z. Xiong, Y. Tian, Z. Fan, Glutamylation of the DNA sensor cGAS regulates its binding and synthase activity in antiviral immunity, Nat. Immunol. 17 (2016) 369-378.

[23] B.P. Kleinstiver, V. Pattanayak, M.S. Prew, S.Q. Tsai, N.T. Nguyen, Z. Zheng, J.K. Joung, High-fidelity CRISPR-Cas9 nucleases with no detectable genome-wide off-target effects, Nature 529 (2016) 490-495.
[24] Y. Fu, J.A. Foden, C. Khayter, M.L. Maeder, D. Reyon, J.K. Joung, J.D. Sander, High-frequency off-target mutagenesis induced by CRISPR-Cas nucleases in human cells, Nat. Biotechnol. 31 (2013) 822-826.

[25] L. Ottini, M. Falchetti, ce:surname>MaederD. Reyon, J.K. Joung, J.D. Sander, High-frequency off-target mutagenesis induced by CRISPR-Cas nucleases in human cells, Nat. Biotechnol. 31 (2013) 822-826.

[25] L. Ottini, M. Falchetti, R. Lupi, P. Rizzolo, V. Agnese, G. Colucci, V. Bazan, A Russo, Patterns of genomic instability in gastric cancer: clinical implications and perspectives, Ann. Oncol. 17 (Suppl. 7) (2006) i97-i102.

[26] A. Menoyo, H. Alazzouzi, E. Espin, M. Armengol, H. Yamamoto, S.J. Schwartz, Somatic mutations in the DNA damage-response genes ATR and CHK1 in sporadic stomach tumors with microsatellite instability, Cancer Res. 61 (2001) 7727-7730.

[27] A. Duval, M. Reperant, A. Compoint, R. Seruca, G.N. Ranzani, B. Jacopetta, R. Hamelin, Target gene mutation profile differs between gastrointestinal and endometrial tumors with mismatch repair deficiency, Cancer Res. 62 (2002) 1609-1612.

[28] S.M. Woerner, A. Benner, C. Sutter, M. Schiller, Y.P. Yuan, G. Keller, P. Bork, M. Doeberitz, J.F. Gebert, Pathogenesis of DNA repair-deficient cancers: a statistical meta-analysis of putative Real Common Target genes, Oncogene 22 (2003) 2226-2235.

[29] A. Staebler, S.F. Lax, L.H. Ellenson, Altered expression of hMLH1 and hMSH2 protein in endometrial carcinomas with microsatellite instability, Hum. Pathol. 31 (2000) 354-358.

[30] S.Q. Tsai, Z. Zheng, N.T. Nguyen, M. Liebers, V.V. Topkar, V. Thapar, N. Wyvekens, C. Khayter, A.J. Iafrate, L.P. Le, M.J. Aryee, J.K. Joung, GUIDE-seq enables genome-wide profiling of off-target cleavage by CRISPR-Cas nucleases, Nat. Biotechnol. 33 (2015) 187-197. 